\title{
Fast neutral outflows in powerful radio galaxies: a major source of feedback in massive galaxies ${ }^{\star}$
}

\author{
R. Morganti ${ }^{1,2}$, C. N. Tadhunter ${ }^{3}$, and T. A. Oosterloo ${ }^{1,2}$ \\ 1 Netherlands Foundation for Research in Astronomy, Postbus 2, 7990 AA, Dwingeloo, The Netherlands \\ e-mail: morganti@astron.nl \\ 2 Kapteyn Astronomical Institute, University of Groningen, PO Box 800, 9700 AV Groningen, The Netherlands \\ 3 Dep. Physics and Astronomy, University of Sheffield, Sheffield, S7 3RH, UK
}

Received 6 September 2005 / Accepted 5 October 2005

ABSTRACT

We report the detection of fast $\left(\sim 1000 \mathrm{~km} \mathrm{~s}^{-1}\right)$, massive outflows of neutral gas observed - using the WSRT - as 21-cm H I absorption against the strong radio continuum of seven radio sources. The neutral outflows occur, in at least somes cases, at kpc distance from the nucleus, and they are most likely driven by the interactions between the expanding radio jets and the gaseous medium enshrouding the central regions. We estimate that the associated mass outflow rates are up to $\sim 50 M_{\odot} \mathrm{yr}^{-1}$, comparable (although at the lower end of the distribution) to the outflow rates found for starburst-driven superwinds in Ultra Luminous IR Galaxies (ULIRG). This suggests that massive, jet-driven outflows of neutral gas in radio-loud AGN can have as large an impact on the evolution of the host galaxies as the outflows associated with starbursts. A radio-loud phase of the AGN is likely a relatively common, albeit short, phase in the life of many (or even all) massive ellipticals. Jet-driven neutral outflows may represent one of the main feedback mechanisms in these galaxies.

Key words. galaxies: active - galaxies: ISM

\section{Introduction: gaseous outflows in AGN}

Galactic mass outflows are spectacular events widely recognised to dramatically affect the evolution of galaxies because of the large amounts of energy they feed back into the interstellar medium. They also appear to be a widespread phenomenon in galaxies, both in the local and in the distant Universe (Heckman 2002; Rupke et al. 2002, 2005a,b; Veilleux et al. 2005; Frey et al. 2002). They can be driven by super-winds associated with large starbursts, or by the energy released by active galactic nuclei (AGN). They have a wide range of effects, from clearing the circum-nuclear regions and halting the growth of the supermassive black-holes (Silk \& Rees 1998; Di Matteo et al. 2005) to injecting energy and metals into the interstellar and intergalactic medium. Indeed, it has been suggested that AGNdriven outflows are a major source of feedback in the overall galaxy formation process, regulating the correlations found between the masses of the central super-massive black-hole and the properties of the host galaxies, and preventing the formation of too many massive galaxies in the early universe. Although numerical simulations demonstrate that AGN-induced outflows are potentially a key component in the evolution of massive

^ Based on observations with the Westerbork Synthesis Radio Telescope. galaxies (Di Matteo et al. 2005), there remains a dearth of hard observational evidence for such outflows and the impact they have on the interstellar medium on the scales of galaxy bulges. Most of the existing evidence for outflows associated with AGN has been obtained using X-ray and UV absorption line observations of the highly ionized gas close to the nucleus, which reveal the presence of broad and narrow absorption line systems that are significantly blueshifted (Crenshaw et al. 2003). However, estimates of scales and physical conditions of such ionised gas outflows are necessarily indirect, their mass outflow rates are difficult to determine, and it is not yet clear whether they are a significant source of feedback on the 1-10 kpc scale of galaxy bulges. Where attempts have been made to characterise the outflows associated with the high ionisation absorption line systems, the derived masses and mass outflow rates are often relatively modest (Crenshaw et al. 2003). Fast, jet-driven outflows of warm, ionised gas have also been detected on a larger 1-100 kpc scale in the extended emission line regions around powerful radio galaxies (Tadhunter 1991; Clark et al. 1998; Villar Martin et al. 1999) but again the masses involved are relatively small, and their impact on the evolution of the host galaxy bulges is unclear.

Here we report the detection of several fast, massive outflows of atomic neutral hydrogen in radio-loud AGN. The 
Table 1. Parameters of the broad H I absorption. The column density is derived assuming $T_{\text {spin }}=1000 \mathrm{~K}$. To estimate the mass outflow rate we used the FWZI/2 of the blueshifted component. References for the systemic velocities: ${ }^{a}$ Holt (2005), ${ }^{b}$ Emonts et al. (2005), ${ }^{c}$ Morganti et al. (2005), ${ }^{d}$ Holt et al. (2003), ${ }^{e}$ Marziani et al. (1993), ${ }^{f}$ Morganti et al. (1998).

\begin{tabular}{lrcccrr}
\hline \hline Name & $\begin{array}{r}V_{\text {sys }} \\
\mathrm{km} \mathrm{s}^{-1}\end{array}$ & $\tau$ & $\begin{array}{c}N_{\mathrm{HI}} \\
10^{21} \mathrm{~cm}^{-2}\end{array}$ & $\begin{array}{c}F W Z I \\
\mathrm{~km} \mathrm{~s}^{-1}\end{array}$ & $\begin{array}{r}\dot{M} \\
M_{\odot} \mathrm{yr}^{-1}\end{array}$ & $\begin{array}{r}r_{*} \\
\mathrm{kpc}\end{array}$ \\
\hline 3C 236 & $30129^{a}$ & 0.0033 & 5 & -1500 & 47 & 0.5 \\
3C 293 & $13450^{b}$ & 0.0038 & 6 & $-1000(+400)$ & 56 & 1 \\
3C 305 & $12550^{c}$ & 0.0023 & 2 & $-500(+150)$ & 12 & 1 \\
3C 459 & $66036^{a}$ & 0.0005 & 0.75 & $-600(+140)$ & 5.5 & 1 \\
4C 12.503 & $36522^{d}$ & 0.0017 & 2.6 & $-1200(+400)$ & $8-21$ & $0.02-0.2$ \\
OQ208 & $22985^{e}$ & 0.0057 & 8.3 & $-1200(+600)$ & 1.2 & 0.01 \\
IC 5063 & $3400^{f}$ & 0.0120 & 10 & $-700(+100)$ & 35 & 0.4 \\
\hline
\end{tabular}

detection of such outflows has been obtained using the unique combination of high sensitivity at $21-\mathrm{cm}$ radio wavelengths and relatively broad bandwidth now available with the upgraded Westerbork Synthesis Radio Telescope (WSRT). We further discuss the impact that they have on the evolution of the host galaxies.

\section{HI outflows: observations and results}

We have used the WSRT to observe a small sample of $11 \mathrm{ob}-$ jects characterised by the presence of a rich ISM surrounding the AGN, e.g. strong CO or far-IR emission, and/or known to have undergone a major episode of star formation in the recent past (Tadhunter et al. 2005) or, as in the case of OQ208, where a Compton-thick medium is pervading the nuclear environment (Guainazzi et al. 2004). Some of them have strong, steep-spectrum core emission (on a scale $<10 \mathrm{kpc}$, unresolved at the resolution of the WSRT $21-\mathrm{cm}$ observations). These objects are considered to be young or recently restarted radio sources.

Unlike previous $\mathrm{H}$ I observations of these galaxies, a wider observing band (20 MHz and 1024 channels) was used. The broad instantaneous band covers $\pm 2000 \mathrm{~km} \mathrm{~s}^{-1}$ around the central velocity corresponding to the frequency of the redshifted H I. This provides a large velocity coverage with sufficient spectral resolution at both sides of the absorptions, important in order to obtain a good subtraction of the continuum. Particular attention has been also paid to the stability of the instrumental spectral response during the observations (typically $12 \mathrm{~h}$ long). The data were calibrated and reduced using the MIRIAD package. The final rms noise is between 0.4 and $0.8 \mathrm{mJy} \mathrm{beam}^{-1}$ and the velocity resolution is about $10 \mathrm{~km} \mathrm{~s}^{-1}$. In each case, the systemic velocity was derived from recent optical spectroscopy observations (see Table 1) and it is indicated in Fig. 1 by a vertical line. The presence of relatively narrow $\left(F W H M<200 \mathrm{~km} \mathrm{~s}^{-1}\right)$ and deep H I absorption was already known for most of the sample objects, but the use of the broad-band has allowed the discovery of the much broader and shallower component of $\mathrm{H}$ I absorption.

In all but one (3C 213.1) of the sources in which an optical depth of a few times $10^{-4}$ could be reached (i.e. the sources with radio core flux $\sim 1 \mathrm{Jy}$ or larger), broad blueshifted H I absorption has been detected. The $\mathrm{HI}$ absorption profiles of the six detected galaxies are shown in Fig. 1. The figure also includes, for completeness, the lower luminosity radio-loud AGN IC 5063 (PKS 2048-57), the first example in which such broad H I was detected (Morganti et al. 1997; Oosterloo et al. 2000) using previous observations with the Australia Telescope Compact Array and LBA. In the case of 3C 213.1, we derive a 3- $\sigma$ upper limit for the optical depth of $\tau=0.0012$, lower than the typical optical depth of the broad absorption listed in Table 1. The remaining three sources (3C 285, 3C 321, 3C 405) of our initial sample have much weaker radio cores (i.e. an optical depth of a few times $10^{-4}$ could not be reached), therefore it is not too surprising that no broad $\mathrm{H}$ I absorption was detected in these objects. A description of the observation and results for the entire sample will be presented in a forthcoming paper.

The characteristics of the broad H I absorption detected are summarized in Table 1 . The broad H I component is typically shallow (peak optical depth as low as $\tau \sim 0.0005$ ), with the full widths at zero intensity $(F W Z I)$ of the absorption ranging between $600 \mathrm{~km} \mathrm{~s}^{-1}$ and almost $2000 \mathrm{~km} \mathrm{~s}^{-1}$ - perhaps the broadest found so far in H I 21-cm absorption in any class of astronomical objects. A large fraction of the H I absorption is blueshifted relative to the systemic velocity of the galaxy. Because the gas producing the absorption must be in front of the radio source, this provides unambiguous evidence that the bulk of gas is outflowing. The typical column densities found for the broad absorption are $1-10 \times 10^{21} \mathrm{~cm}^{-2}$ (see Table 1). These values for the column density have been estimated using $T_{\text {spin }}=1000 \mathrm{~K}$. This value is likely to be this high (or even higher, Tadhunter et al. in prep.) because of the extreme conditions of the gas, the proximity to the active nucleus (Bahcall \& Ekers 1969) and the presence of shocks. The FWZI are given with respect to the systemic velocity. It is clear that most of the H I absorption is blueshifted, although a redshifted component (given in parenthesis) is also observed in almost all the objects. In some cases we know that this component is coming from regularly rotating gas in the host galaxies (Morganti et al. 2005).

The low optical depth of the broad H I absorption features makes them difficult to detect using present day radio telescopes, even if they have the sensitivity and bandwidth of the WSRT. However, the high detection rate in the selected objects suggests that the broad Hi phenomenon is not exceptional, although it might be connected to a particular stage in the 


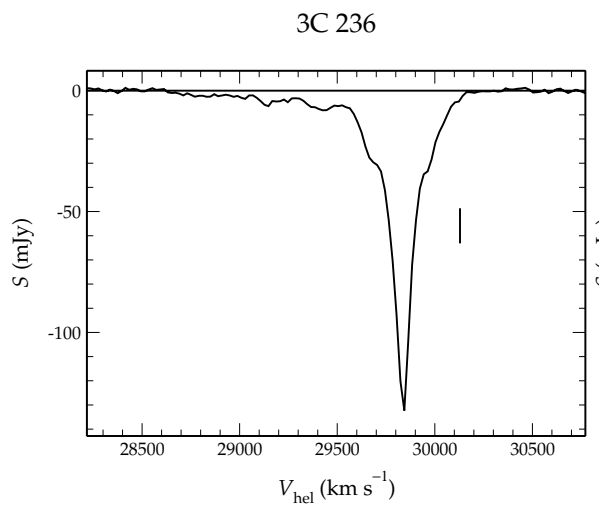

3C 459

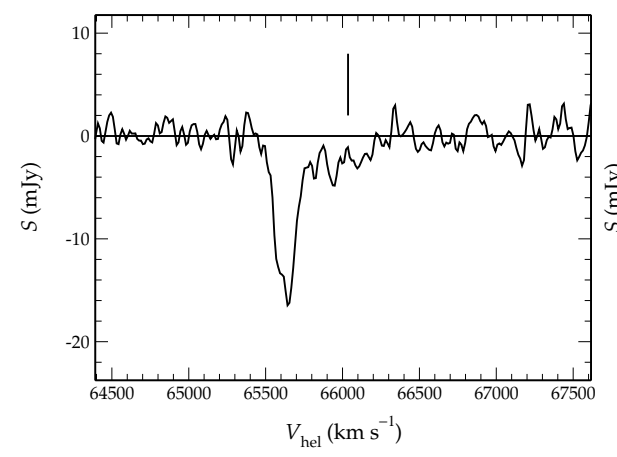

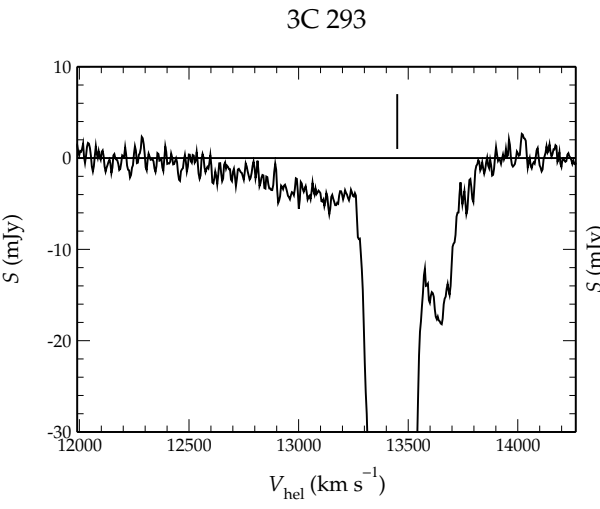

OQ 208

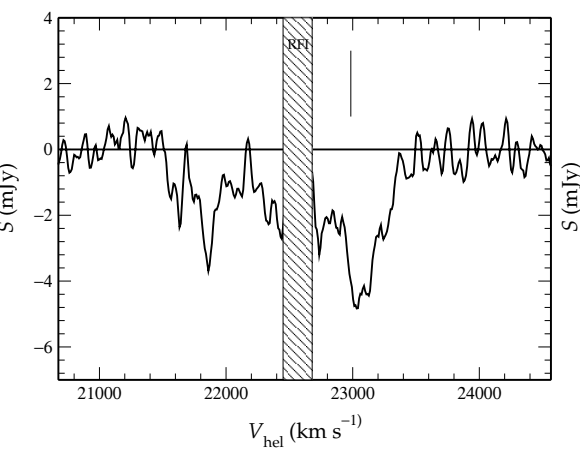

IC 5063

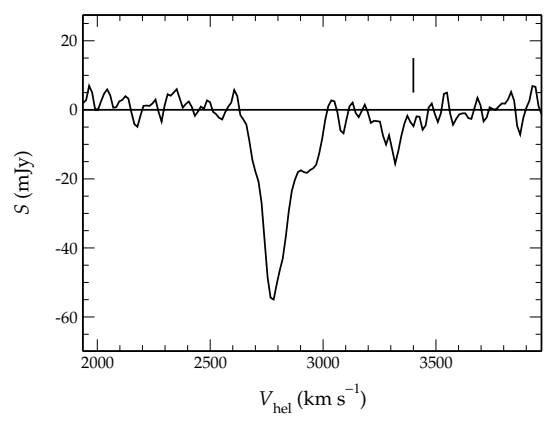

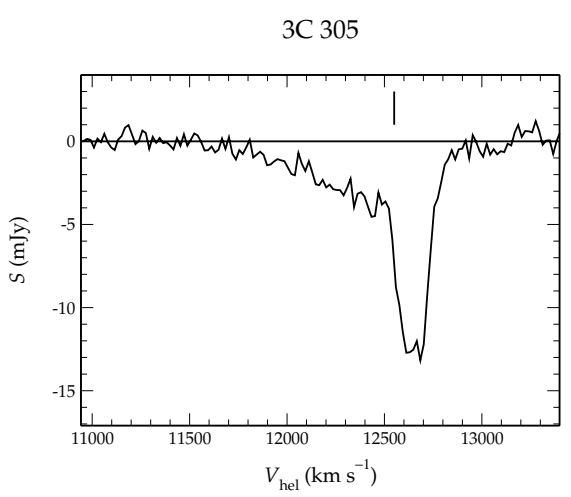

$4 \mathrm{C} 12.50$

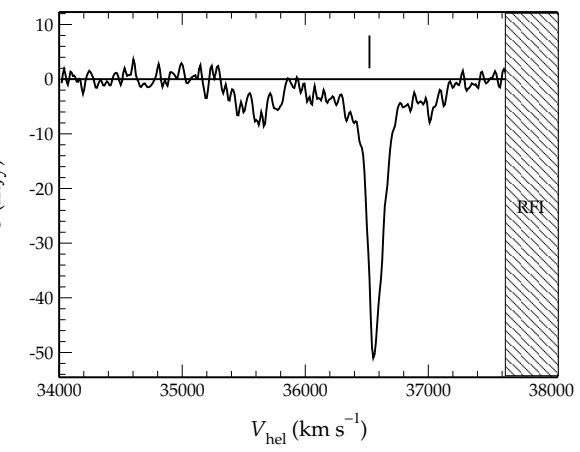

Fig. 1. $21 \mathrm{~cm}-\mathrm{HI}$ absorption profiles detected against seven radio-loud AGN. The observations of the radio galaxies were done using the upgraded WSRT. The broad H I absorption in the lower luminosity radio-loud AGN IC 5063 (PKS 2048-57) (detected using the LBA, Oosterloo et al. 2000) is shown for completeness. The vertical line indicates the systemic velocity.

evolution of the galaxy, for example, the early phases of the expansion of the radio jets through the bulges of the host galaxies. Indeed, it is intriguing that all the fast $\mathrm{H}$ I outflows have been detected so far are in radio galaxies that are in the initial (or restarted) phase of their radio activity.

\section{Origin and mass outflow rates}

Different mechanisms have been proposed to explain AGNdriven outflows (Tadhunter et al. 2001; Elvis et al. 2002; Dopita et al. 2002). In the case of the fast H I outflows presented here, there is now strong evidence that the most likely mechanism is the interaction between radio jets and the surrounding inter stellar medium. This evidence comprises follow-up observations at higher resolution of two of the objects (IC 5063 and $3 \mathrm{C} 305$ ) in our sample that demonstrate that the outflow regions are resolved on a scale of $200 \mathrm{pc}-1.6 \mathrm{kpc}$, and spatially associated with both the extended radio knots along the jets and the warm gas outflows detected at the same locations in optical observations (Oosterloo et al. 2000; Morganti et al. 2005). In a third case (3C 293), the evidence that the outflow is located at $\sim 1 \mathrm{kpc}$ is more indirect (Morganti et al. 2003; Emonts et al. 2005). The presence of neutral atomic gas accelerated to such high velocities is in itself intriguing. It indicates that after a strong jet-cloud interaction the gas can cool very efficiently. Such rapid cooling is indeed predicted by recent numerical simulations of jets impacting on gas clouds (Mellema et al. 2002; Fragile et al. 2004). Fast outflows of ionised gas are also detected using optical emission lines in all the galaxies in Table 1 for which good optical spectra are available (Holt et al. 2003, 2005; Morganti et al. 2005; Emonts et al. 2005). The presence of ionised gas can be explained quite naturally as result of the shock produced by the radio plasma interacting with the ISM. The similarities found between the kinematics 
of the neutral and ionised gas indicate that the two phases of the gas are part of the same outflow. However, the outflows of ionised gas are typically much less massive (Morganti et al. 2005; Emonts et al. 2005) than those of the neutral hydrogen. The mass outflow rate has been calculated following Heckman (2002) and Rupke et al. (2002):

$$
\dot{M}=30 \cdot \frac{\Omega}{4 \pi} \cdot \frac{r_{*}}{1 \mathrm{kpc}} \cdot \frac{N_{\mathrm{H}}}{10^{21} \mathrm{~cm}^{-2}} \cdot \frac{v}{300 \mathrm{kms}^{-1}} M_{\odot} \mathrm{yr}^{-1}
$$

where the mass is flowing at a velocity $v$ (for which we used the $F W Z I / 2$ of the blueshifted component) into a solid angle $\Omega$ assumed to be $\pi$ steradians from a radius $r_{*}$, indicated in the last column. These values have been obtained for three galaxies from high resolution follow-up observations (Oosterloo et al. 2000; Morganti et al. 2005; Emonts et al. 2005). For the others sources we assumed that the broad absorption comes from the region with the stronger radio continuum that, in these objects, is usually not coincident with the radio core, but with blobs along the radio jet or lobe-like structures. One should also take into account that the $\mathrm{HI}$ absorption can be traced only where the background continuum is observed, therefore the region of the outflow may be much larger.

The derived values, ranging from $\sim 1$ to over $50 M_{\odot} \mathrm{yr}^{-1}$ (see Table 1), are remarkable. They are higher than most of the mass outflow rates deduced for ionized gas using X-ray and UV absorption line measurements of nearby AGN (Crewshaw et al. 2003), although in some nearby Seyfert galaxies value up to $10 M_{\odot} \mathrm{yr}^{-1}$ have been also derived (Veilleux et al. 2005). The values we derive for the neutral hydrogen outflows, correspond to the lower end of the distribution for starburst super-winds in Ultra-Luminous IR Galaxies (ULIG) that show outflows rates (Heckman 2002; Rupke et al. 2002, 2005a,b) ranging from 10 to $1000 M_{\odot} \mathrm{yr}^{-1}$.

\section{Implications for the evolution of the host galaxies}

Starburst super-winds are recognized to be responsible for inhibiting early star formation, enriching the ICM with metals and heating the ISM/IGM medium. Given the mass outflow rates obtained above, the results presented here show that similar effects could be produced by the jet during the radio-loud phase of AGN activity. Indeed, the jet in powerful radio galaxies (as those considered in this work) can be extremely energetic $\left(\gtrsim 10^{58} \mathrm{erg}\right.$, when integrated over the lifetime of the radio source), often exceeding the gravitational binding energy of the gas in a typical galaxy, and only a fraction of the power of the jets is needed to produce the H I outflows.

More in general, to investigate the importance of the $\mathrm{HI}$ outflows, we can compare the the bulk energy of the outflow with the typical binding energy of the gas in the galaxy. Assuming a typical galaxy with a gas mass of $M_{\text {gas }} \sim 1 \times$ $10^{10} M_{\odot}$, at a typical radius of $r_{\mathrm{g}} \sim 5 \mathrm{kpc}$, and a total mass within $5 \mathrm{kpc}$ of $M_{\mathrm{gal}} \sim 10^{11} M_{\odot}$, the binding energy is $\sim 2 \times$ $10^{58}$ erg. For comparison, a $10 M_{\odot} \mathrm{yr}^{-1}$ outflow moving outwards at $500 \mathrm{~km} \mathrm{~s}^{-1}$ has a bulk kinetic energy of $\sim 2.5 \times 10^{57} \mathrm{erg}$ (assuming that the outflow is integrated over $10^{8} \mathrm{yr}$ lifetime of the radio source). This value could easily be increased by a factor of two if we take into account the turbulent motion (indeed the H I absorption lines are as broad as they are shifted). Thus, the kinetic power in the H I outflows can represent a substantial fraction of the energy required to remove the gas from the bulges of the galaxies, and, therefore, can provide an important source of feedback.

The massive HI outflows show, more clearly than optical emission line studies (Tadhunter et al. 2001), that AGN have a major effect on the circumnuclear gas; the central kiloparsec region of AGN host galaxies are clearly dynamic places with the distribution of ISM evolving strongly as a function of time. Our results also have implications for the evolution of the radio sources. Although, as mentioned above, the radio jets in these galaxies are unlikely to be completely confined by the surrounding dense interstellar medium, the strong interaction that we detect through the kinematic signature of the broad $\mathrm{H} \mathrm{I}$ can disrupt the paths of the jets and slow down their overall expansion, as predicted in numerical simulations (Morganti et al. 2004; Saxton et al. 2005). This effect is likely to be even more relevant in higher-density conditions prevailing in the early universe.

To date, the majority of studies of atomic neutral hydrogen in radio galaxies have been made using a relatively narrow bandwidth and low sensitivity. This automatically limits the possibility of detecting $\mathrm{H}$ I with the extreme kinematics described here. Although the detection of this component is complicated by the low optical depth, future observations extended to larger samples of both compact and more extended radio galaxies will reveal whether this phenomenon is significant at all stages of evolution of powerful, radio-loud AGN.

Acknowledgements. The Westerbork Synthesis Radio telescope is operated by the ASTRON (Netherlands Foundation for Research in Astronomy) with support of The Netherlands Foundation for Scientific Research (NWO).

\section{References}

Bahcall J. N., \& Ekers R. D. 1969, ApJ, 157, 1055

Clark, N. E., Axon, D. J., Tadhunter, C. N., Robinson, A., \& O'Brien, P. 1998, ApJ, 494, 546

Crenshaw, D. M., Kraemer S. B., \& George I. M. 2003, ARA\&A, 41, 117

Di Matteo, T., Springel V., \& Hernquist L. 2005, Nature, 433, 604

Dopita, M. A., Groves B. A., Sutherland R. S., Binette L., \& Cecil, G. 2002, ApJ, 572, 753

Elvis, M., Marengo M., \& Karovska M. 2002, ApJ, 567, L107

Emonts, B. H. C., Morganti, R., Tadhunter, C. N., et al. 2005, MNRAS, in press

Fragile, C. P., Murray, S. D., Anninos, P., \& van Breugel, W. 2004, ApJ, 604, 74

Frye, B., Broadhurst, T., \& Benitez, N. 2002, ApJ, 568, 558

Guainazzi, M., Siemiginowska, A., Rodriguez-Pascual, P., \& Stanghellini, C. 2004, A\&A, 421, 461

Heckman, T. M. 2002, in Extragalactic Gas at Low Redshift, ed. J. Mulchay, \& J. Stoke, ASP Conf. Ser., 254, 292

[arXiv:astro-ph/0107438)] 
Holt, J., Tadhunter, C. N., \& Morganti, R. 2003, MNRAS, 342, 227 Holt, J. 2005, Ph.D. Thesis, University of Sheffield

Marziani, P., Sulentic, J. W., Calvani, M., et al. 1993, ApJ, 410, 56 Mellema, G., Kurk J. D., \& Rottgering, H. J. A. 2002, A\&A, 395, L13

Morganti, R., Oosterloo T., \& Tsvetanov, Z. 1998, AJ, 115, 915

Morganti, R., Oosterloo, T. A., Emonts, B. H. C., van der Hulst, J. M., \& Tadhunter, C. N. 2003, ApJ, 593, L69

Morganti, R., Oosterloo, T. A., Tadhunter, C. N., et al. 2004, A\&A, 424, 119

Morganti, R., Oosterloo, T. A., Tadhunter, C. N., van Moorsel, G., \& Emonts, B. 2005, A\&A, 439, 521

Oosterloo, T. A., Morganti, R., Tzioumis, A., et al. 2000, AJ, 119, 2085

Rupke, D. S., Veilleux S., \& Sanders, D. B. 2002, ApJ, 570, 588
Rupke, D. S., Veilleux S., \& Sanders, D. B. 2005a, ApJS, 160, 87 Rupke, D. S., Veilleux S., \& Sanders, D. B. 2005b, ApJS, 160, 115 Saxton C. J., Bicknell G., Sutherland R. S., \& Midgley, S. 2005, MNRAS, 359, 781

Silk, J., \& Rees, M. J. 1998, A\&A, 331, L1

Tadhunter, C. N. 1991, MNRAS, 251, 46

Tadhunter, C., Wills K., Morganti R., Oosterloo T., \& Dickson, R. 2001, MNRAS, 327, 227

Tadhunter, C. N., Robinson, T. G., Gonzalez Delgano, R. M., Wills, K., \& Morganti, R. 2005, MNRAS, 356, 480

Veilleux, S., Cecil G., \& Bland-Hawthorn, J. 2005, ARA\&A, in press [arxiv:astro-ph/0504435]

Villar-Martin, M., Tadhunter, C., Morganti, R., Axon, D., \& Koekemoer, A. 1999, MNRAS, 307, 24 